International Journal of Pure and Applied Mathematics

Volume 107 No. 2 2016, 407-414

ISSN: 1311-8080 (printed version); ISSN: 1314-3395 (on-line version)

url: http://www.ijpam.eu

doi: 10.12732/ijpam.v107i2.10

\title{
EXISTENCE AND UNIQUENESS OF WEAK SOLUTION OF A NONLINEAR NEUMANN PROBLEM
}

\author{
Bassam Al-Hamzah $^{1}$, Naji Yebari ${ }^{2}$ \\ ${ }^{1,2}$ Department of Mathematics \\ Faculty of Sciences \\ University Abdelmalek Essaadi \\ Tetouan, MOROCCO
}

\begin{abstract}
This paper deals with the equation

$$
-\Delta_{p} u+a(x)|u|^{p-2} u=f(x, u)
$$
laplacian boundary value of the form

$$
\left\{\begin{array}{lr}
-\Delta_{p} u+a(x)|u|^{p-2} u=f(x, u), & \text { in } \Omega, \\
\frac{\partial u}{\partial \eta}=0, & \text { on } \partial \Omega
\end{array}\right.
$$
\end{abstract}

in bounded domain $\Omega \in \mathbb{R}^{N}$. Relying on Browder theorem, under conditions of the monotonous function $f$. We obtained the existence and uniqueness of weak solutions for the weighted p-

in bounded domain $\Omega \in \mathbb{R}^{N}$.

Key Words: weak solutions, p-Laplacian operator, Neumann problem

\section{Introduction}

In this paper, we are concerned with the existence and uniqueness of weak solution for a weighted p-laplacian boundary value of the form:

$$
\left\{\begin{array}{lr}
-\Delta_{p} u+a(x)|u|^{p-2} u=f(x, u), & \text { in } \Omega, \\
\frac{\partial u}{\partial \eta}=0, & \text { on } \partial \Omega .
\end{array}\right.
$$

Let $\Omega$ be a bounded domain in $\mathbb{R}^{N}$ and $f: \Omega \times \mathbb{R} \rightarrow \mathbb{R}$ be a Caratheodry (CAR) Satisfies the following:

Received: January 4, 2016

Published: April 9, 2016

$\S$ Correspondence author
(C) 2016 Academic Publications, Ltd.

url: www.acadpubl.eu 
$\left(H_{1}\right) f\left(x, s_{1}\right) \leq f\left(x, s_{2}\right)$ for a.e. $x \in \Omega$ and $\forall s_{1}, s_{2} \in \mathbb{R}, s_{1} \geq s_{2}$.

$\left(H_{2}\right)|f(x, s)| \leq f_{0}(x)+c|s|^{p-1}$ p.p. $x \in \Omega s \in \mathbb{R}$ that there exists $f_{0} \in$ $L^{p^{\prime}}(\Omega)$, and $c>0$.

$\left(H_{3}\right) 0<\alpha \leq a(x) \leq \beta<+\infty$.

This paper is organized as follows: Section 2 contains some basic definitions concerning the nonlinear operators that will be used throughout the paper. Also, we introduce the space setting of the problem and give some basic characteristics, as the equivalent norm and embedding results. In Section 3 we state the main result on the existence and uniqueness of weak solutions of the problem $(\mathrm{P})$.

\section{Preliminaries and Space Setting}

First, we introduce some basic definitions concerning the nonlinear operators which we use extensively in this paper (0.3).

Definition 2.1. (see [7]) Let $A: V \rightarrow V^{\prime}$ be an operator on a real Banach space $V$. We say that the operator $A$ is:

(i) bounded iff it maps bounded sets into bounded i.e. for each $r>0$ there exists $M>0$ ( $M$ depending on $r$ ) such that

$$
\|u\| \leq r \Rightarrow\|A(u)\| \leq M, \forall u \in V
$$

(ii) coercive: iff

$$
\lim _{\|u\| \rightarrow \infty} \frac{\langle A(u), u\rangle}{\|u\|}=\infty ;
$$

(iii) monotone iff $\left\langle A\left(u_{1}\right)-A\left(u_{2}\right), u_{1}-u_{2}\right\rangle \geq 0, \forall u_{1}, u_{2} \in V$;

(iv) strictly monotone iff

$$
\left\langle A\left(u_{1}\right)-A\left(u_{2}\right), u_{1}-u_{2}\right\rangle>0, \text { for all } u_{1}, u_{2} \in V, u_{1} \neq u_{2} ;
$$

(v) strongly monotone iff there exists $k>0$,

$$
\left\langle A\left(u_{1}\right)-A\left(u_{2}\right), u_{1}-u_{2}\right\rangle \geq k\left\|u_{1}-u_{2}\right\|, \text { for all } u_{1}, u_{2} \in V, u_{1} \neq u_{2} ;
$$

(vi)continuous iff $u_{n} \rightarrow u$ implies $A\left(u_{n}\right) \rightarrow A(u)$, for all $u_{n}, u \in V$;

(vii) strongly continuous iff $u_{n} \rightarrow u$ implies $A\left(u_{n}\right) \rightarrow A(u)$, for all $u_{n}, u \in V$;

(viii) demicontinuous iff $u_{n} \rightarrow u$ implies $A\left(u_{n}\right) \rightarrow A(u)$, for all $u_{n}, u \in V$. 
Theorem 2.1. (Browder, see [7]) Let $A$ be a reflexive real Banach space. Moreover let $A: V \rightarrow V^{\prime}$ be an operator which is: bounded, demicontinuous, coercive, and monotone on the space $V$. Then, the equation $A(u)=f$ has at least one solution $u \in V$ for each $f \in V^{\prime}$.

If moreover, $A$ is strictly monotone operator, then the equation $(P)$ has precisely one solution $u \in V$ for every $f \in V^{\prime}$.

\section{Existence and Uniqueness Results}

In this section, using Browder theorem, we prove the existence and uniqueness of weak solution for equation $(\mathrm{P})$.

Definition 3.1. We say that $u \in W^{1, p}(\Omega)$ is a weak solution to equation $(P)$ if

$$
\int_{\Omega}|\nabla u|^{p-2} \nabla u \nabla \varphi d x+\int_{\Omega} a(x)|u|^{p-2} u \varphi d x=\int_{\Omega} f(x, u) \varphi d x, \forall \varphi \in W^{1, p}(\Omega) .
$$

Our main results concerning problem $(\mathrm{P})$ is the following theorem.

Theorem 3.1. Let $p \geq 2$, and $f(x, u) \in C A R(\Omega, \mathbb{R})$ satisfy $\left(H_{1}\right),\left(H_{2}\right)$ and $\left(H_{3}\right)$. Then problem $(P)$ has a unique weak solution.

Proof. We define for the operator $A: W^{1, p}(\Omega) \rightarrow\left(W^{1, p}(\Omega)\right)^{\prime}$, as $A=J-F$, where the Operators $J: W^{1, p}(\Omega) \rightarrow\left(W^{1, p}(\Omega)\right)^{\prime}$, and $F: W^{1, p}(\Omega) \rightarrow\left(W^{1, p}(\Omega)\right)^{\prime}$ are given by

$$
\langle J(u), \varphi\rangle=\int_{\Omega}|\nabla u|^{p-2} \nabla u \nabla \varphi d x+\int_{\Omega} a(x)|u|^{p-2} u \varphi d x
$$

and

$$
\langle F(u), \varphi\rangle=\int_{\Omega} f(x, u) \varphi d x
$$

for all $u, \varphi \in W^{1, p}(\Omega)$. Thus, to find a weak solution of $(P)$ is equivalent to finding $W^{1, p}(\Omega)$ which satisfies the operator equation $A(u)=0$. Now, we have the following properties of the operators $J$ and $F$.

a) $J$ and $F$ are well defined. Using Holders inequality, we have

$$
\langle J(u), \varphi\rangle=\int_{\Omega}|\nabla u|^{p-2} \nabla u \nabla \varphi d x+\int_{\Omega} a(x)|u|^{p-2} u \varphi d x,
$$




$$
\begin{aligned}
|\langle J(u), \varphi\rangle| & \leq \int_{\Omega}|\nabla u|^{p-1}|\nabla \varphi| d x+\int_{\Omega} a(x)|u|^{p-1}|\varphi| \\
& \leq\left(\int_{\Omega}|\nabla u|^{p}\right)^{\frac{1}{p^{\prime}}}\left(\int_{\Omega}|\nabla \varphi|^{p}\right)^{\frac{1}{p}}+\beta\left(\int_{\Omega}|u|^{p}\right)^{\frac{1}{p^{\prime}}}\left(\int_{\Omega}|\varphi|^{p}\right)^{\frac{1}{p}}<\infty .
\end{aligned}
$$

Moreover

$$
\langle F(u), \varphi\rangle=\int_{\Omega} f(x, u) \varphi d x
$$

and

$$
|\langle F(u), \varphi\rangle| \leq \int_{\Omega}|f(x, u) \varphi| d x \leq \int_{\Omega}\left(f_{0}(x)+c|u|^{p-1}\right)|\varphi| d x .
$$

By Hölder's inequality,

$$
|\langle F(u), \varphi\rangle| \leq\left(\int_{\Omega}\left|f_{0}(x)\right|^{p^{\prime}}\right)^{\frac{1}{p^{\prime}}}\left(\int_{\Omega}|\varphi|^{p}\right)^{\frac{1}{p}}+c\left(\int_{\Omega}|u|^{p}\right)^{\frac{1}{p^{\prime}}}\left(\int_{\Omega}|\varphi|^{p}\right)^{\frac{1}{p}}<\infty .
$$

Hence $J, F$ are well defined.

b) $J$ and $F$ are bounded operators. Indeed, for every u such that $\|u\|_{W^{1, p}(\Omega)}$ $\leq M$,

$$
\begin{gathered}
\|J(u)\|_{\left(W^{1, p}(\Omega)\right)^{\prime}}=\sup _{\|\varphi\|_{W^{1, p}(\Omega)} \leq 1}|\langle J(u), \varphi\rangle| \\
\left.\leq \sup _{\|\varphi\|_{W^{1, p}(\Omega)} \leq 1}\left(\int_{\Omega}|\nabla u|^{p}\right)^{\frac{1}{p^{\prime}}}\left(\int_{\Omega}|\nabla \varphi|^{p}\right)^{\frac{1}{p}}+\beta\left(\int_{\Omega}|u|^{p}\right)^{\frac{1}{p^{\prime}}}\left(\int_{\Omega}|\varphi|^{p}\right)^{\frac{1}{p}}\right) \\
=\sup _{\|\varphi\| \leq 1}\left(\|\nabla u\|_{L^{p}}^{\frac{p}{p^{\prime}}}\|\varphi\|_{L^{p}}+\beta\|u\|_{L^{p}}^{\frac{p}{p^{\prime}}}\|\varphi\|_{L^{p}}\right) \\
\leq\|\nabla u\|_{L^{p}}^{\frac{p}{p^{\prime}}}+\beta\|u\|_{L^{p}}^{\frac{p}{p^{\prime}}} \leq\|u\|^{\frac{p}{p^{\prime}}}+\beta\|u\|^{\frac{p}{p^{\prime}}} \\
2 \beta\|u\|^{\frac{p}{p^{\prime}}} \leq 2 \beta M^{\frac{p}{p^{\prime}}} .
\end{gathered}
$$

Also, we get

$$
\begin{gathered}
\|F(u)\|_{\left(W^{1, p}(\Omega)\right)^{\prime}}=\sup _{\|\varphi\|_{W^{1, p}(\Omega)} \leq 1}|\langle F(u), \varphi\rangle| \leq \sup _{\|\varphi\|_{W^{1, p}(\Omega)} \leq 1} \int_{\Omega}\left(f_{0}(x)+c|u|^{p-1}\right)|\varphi| \\
\quad \leq \sup _{\|\varphi\|_{W^{1, p}(\Omega)} \leq 1}\left[\left(\int_{\Omega}\left|f_{0}(x)\right|^{p^{\prime}}\right)^{\frac{1}{p^{\prime}}}+\left(\int_{\Omega}|u|^{(p-1) p^{\prime}}\right)^{\frac{1}{p^{\prime}}}\right]\left(\int_{\Omega}|\varphi|^{p}\right)^{\frac{1}{p}} .
\end{gathered}
$$




$$
\begin{gathered}
\leq k\left(\left\|f_{0}\right\|_{L^{p^{\prime}}(\Omega)}+k\|u\|_{W^{1, p}(\Omega)}^{\frac{p}{p^{\prime}}}\right) \\
\leq k\left(\left\|f_{0}\right\|_{L^{p^{\prime}(\Omega)}}+k M^{\frac{p}{p^{\prime}}}\right)
\end{gathered}
$$

Where $\mathrm{k}$ is the constant of the embedding of $W^{1, p}(\Omega)$ into $L^{p}(\partial \Omega)$,

c) $J$ and $F$ are continuous operators if $u_{n} \rightarrow u$, in $W^{1, p}(\Omega)$. Then, we have

$$
\left\|u_{n}-u\right\|_{W^{1, p}(\Omega)} \rightarrow 0,\left\|\nabla u_{n}-\nabla u\right\|_{L^{p}(\Omega)} \rightarrow 0,\left\|u_{n}-u\right\|_{L^{p}} \rightarrow 0
$$

Applying Dominated Convergence Theorem, we obtain

$$
\left\|\left(\left|\nabla u_{n}\right|^{p-2} \nabla u_{n}-|\nabla u|^{p-2} \nabla u\right)\right\|_{L^{p}(\Omega)} \rightarrow 0
$$

and

$$
\left\|\left(\left|u_{n}\right|^{p-2} u_{n}-|u|^{p-2} u\right)\right\|_{L^{p}(\Omega)} \rightarrow 0
$$

Hence

$$
\begin{gathered}
\left\|J\left(u_{n}\right)-J(u)\right\|_{\left(W^{1, p}(\Omega)\right)^{\prime}}=\sup _{\|\varphi\|_{W^{1, p}(\Omega)} \leq 1}\left|\left\langle J\left(u_{n}\right)-J(u), \varphi\right\rangle\right| \\
\leq \sup _{\|\varphi\|_{W^{1, p}(\Omega)} \leq 1}\left(\left(\int_{\Omega}\left(\left[\left|\nabla u_{n}\right|^{p-2} \nabla u_{n}-|\nabla u|^{p-2} \nabla u\right]\right)^{p^{\prime}}\right)^{\frac{1}{p^{\prime}}}\left(\int_{\Omega}|\varphi|^{p}\right)^{\frac{1}{p}}\right. \\
\left.+\beta\left(\int_{\Omega}\left(\left(\left[\left|u_{n}\right|^{p-2} u_{n}-|u|^{p-2} u\right)\right]\right)^{p^{\prime}}\right)^{\frac{1}{p^{\prime}}}\left(\int_{\Omega}|\varphi|^{p}\right)^{\frac{1}{p}}\right) \rightarrow 0 \text { as } n \rightarrow \infty .
\end{gathered}
$$

Similarly, we have

$$
\begin{gathered}
\left\|F\left(u_{n}\right)-F(u)\right\|_{\left(W^{1, p}(\Omega)\right)^{\prime}}=\sup _{\|\varphi\|_{W^{1, p}(\Omega)} \leq 1}\left|\left\langle F\left(u_{n}\right)-F(u), \varphi\right\rangle\right| \\
\leq k\left(\int_{\Omega}\left|f\left(x, u_{n}\right)-f(x, u)\right|^{p^{\prime}}\right)^{\frac{1}{p^{\prime}}} \rightarrow 0 \text { as } n \rightarrow \infty .
\end{gathered}
$$

d) Let $p \geq 2, \forall x_{1}, x_{2} \in \mathbb{R}^{\mathbb{N}}$, we have the following inequality

$$
\left|x_{2}\right|^{p} \geq\left|x_{1}\right|^{p}+p\left|x_{1}\right|^{p-2} x_{1}\left(x_{2}-x_{1}\right)+\frac{\left|x_{2}-x_{1}\right|^{p}}{2^{p-1}-1} .
$$

Now,

$$
\langle J(u)-J(\varphi), u-\varphi\rangle=\int_{\Omega}\left[|\nabla u|^{p-2} \nabla u-|\nabla \varphi|^{p-2} \nabla \varphi\right](\nabla u-\nabla \varphi)
$$




$$
\begin{gathered}
+\int_{\Omega} a(x)\left[|u|^{p-2} u-|\varphi|^{p-2} \varphi\right](u-\varphi) \\
=\int_{\Omega}\left[|\nabla u|^{p-2} \nabla u(\nabla u-\nabla \varphi)\right]-\int_{\Omega}\left[|\nabla \varphi|^{p-2} \nabla \varphi(\nabla u-\nabla \varphi)\right] \\
+\int_{\Omega} a(x)\left[|u|^{p-2} u(u-\varphi)\right]-\int_{\Omega} a(x)\left[|\varphi|^{p-2} \varphi(u-\varphi)\right]=I_{1}+I_{2} .
\end{gathered}
$$

Using (1), we get

$$
\begin{gathered}
I_{1}+I_{2} \geq \frac{2}{p\left(2^{p-1}-1\right)} \int_{\Omega}|\nabla u-\nabla \varphi|^{p} d x+\frac{2}{p\left(2^{p-1}-1\right)} \int_{\Omega} a(x)|u-\varphi|^{p} d x \\
\geq \alpha c(p)\left(\|\nabla u-\nabla \varphi\|_{L^{p}(\Omega)}^{p}+\|u-\varphi\|_{L^{p}(\Omega)}^{p}\right) \\
=\alpha c(p)\|u-\varphi\|_{W^{1, p}(\Omega)}^{p}, \text { for } p \geq 2 .
\end{gathered}
$$

So

$$
\langle J(u)-J(\varphi), u-\varphi\rangle \geq \alpha c(p)\|u-\varphi\|_{W^{1, p}(\Omega)}^{p} \text { for } p \geq 2 .
$$

Also, we get

$$
\langle F(u)-F(\varphi), u-\varphi\rangle=\int_{\Omega}[f(x, u)-f(x, \varphi)](u-\varphi) .
$$

Since $f$ is decreasing with respect to the second variable, we have

$$
[f(x, u)-f(x, \varphi)](u-\varphi) \leq 0 .
$$

Consequently

$$
\langle F(u)-F(\varphi), u-\varphi\rangle=\int_{\Omega}[f(x, u)-f(x, \varphi)](u-\varphi) \leq 0 .
$$

Equations (2) and (3) imply that

$$
\langle A(u)-A(\varphi), u-\varphi\rangle \geq c(p)\|u-\varphi\|_{W^{1, p}(\Omega)}^{p} \text { for } p \geq 2 .
$$

So $A$ is strongly monotone. Now, to apply Browder theorem, it remains to prove that A is a coercive operator. From (4), we have

$$
\langle A(u), u\rangle \geq\langle A(0), u\rangle+c(p)\|u\|_{W^{1, p}(\Omega)}^{p} .
$$

On the other hand

$$
\langle A(0), u\rangle=\langle J(0), u\rangle-\langle F(0), u\rangle
$$




$$
\begin{gathered}
=-\int_{\Omega} f(x, 0) u \\
\geq-\left(\int_{\Omega}\left[f_{0}(x)\right]^{p^{\prime}}\right)^{\frac{1}{p^{\prime}}}\left(\int_{\Omega}|u|^{p}\right)^{\frac{1}{p}} \\
\geq-k\left\|f_{0}\right\|_{L^{p^{\prime}}(\Omega)}\|u\|_{W^{1, p}(\Omega)} .
\end{gathered}
$$

Then

$$
\langle A(u), u\rangle \geq c(p)\|u\|_{W^{1, p}(\Omega)}^{p}-k\left\|f_{0}\right\|_{L^{p^{\prime}}(\Omega)}\|u\|_{W^{1, p}(\Omega)} .
$$

So

$$
\lim _{\|u\|_{W^{1, p}(\Omega)} \rightarrow \infty} \frac{\langle A(u), u\rangle}{\|u\|_{W^{1, p}(\Omega)}}=\infty .
$$

This proves the coercivity condition and so, the existence of weak solution for $(\mathrm{P})$. The uniqueness of weak solution of $(\mathrm{P})$ is a direct consequence of $(4)$. Suppose that $u, \varphi$ be a weak solutions of $(\mathrm{P})$ such that $u \neq \varphi$. Now, from (4), we have

$$
0=\langle A(u)-A(\varphi), u-\varphi\rangle \geq c(p)\|u-\varphi\|_{W^{1, p}(\Omega)}^{p} \geq 0 .
$$

Therefore $u=\varphi$ This completes the proof.

\section{References}

[1] G. A. Afrouzi, S. Mahdavi, Z. Naghizadeh, Existence and Uniqueness of Solution for P Laplacian Dirichlet Problem, ISSN 1749-3889 (print), 1749-3897 (online), International Journal of Nonlinear Science, Vol. 8(2009) No. 3, pp. 274-278, (Received 6 November 2008, accepted 15 July 2009).

[2] R. A. Hameed, J. Sun, B. Wu, Existence of periodic solutions of a p-Laplacian-Neumann problem, a Springer open Journal, Boundary Value Problems 2013, 2013:171.

[3] B. Al Hamzah, N. Yebari, Existence and uniqueness of weak solution for weighted pLaplacian Steklov problem. International Journal of Innovation and Applied Studies, Vol. 11 No. 1 Apr. 2015, pp. 69-76.

[4] K. Bohmer, On finite element methods for fully nonlinear elliptic equations of second order, SIAM Journal on Numerical Analysis 46 (2008) 12121249.

[5] S. De Lillo, M. Sommacal, Neumann problem on the semi-line for the Burgers equation. a Springer open Journal, Boundary Value Problems 2011, 2011:34.

[6] A.R. El Amrouss, S. El Habib, N. Tsouli, Existence of solutions for an eigenvalue problem with weight. Electronic Journal of Differential Equations, Vol. 2010(2010), No. 45, pp. 110. ISSN: 1072-6691.

[7] S. A. Khafagy, Existence and uniqueness of weak solution for weighted p-Laplacian Dirichlet problem, Vol. 3, 2011, Online ISSN 1943-023X, Jouranal of Advanced Research in Dynamical and Control Systems. 
[8] S. A. Khafagy, Existence and Uniqueness of Weak Solution for Quasilinear Elliptic (p, q)-Laplacian System. Global Journal of Pure and Applied Mathematics. ISSN0973-1768 Volume 8, Number 4 (2012), pp. 465475.

[9] A. El Khalil, S. Kellati A. Touzani, On the spectrum of the p-biharmonic operator. 2002-Fez conference on Partial Dikerential Equations, Electronic Journal of Dikeren- tial Equations, Conference 09, 2002, pp 161170.

[10] A. Ferrero, F. Gazzola, and T. Weth, On a fourth order Steklov eigenvalue problem, Analysis, 25, 315332 (2005).

[11] M. Kumar, P. Kumar, Simulation of a nonlinear Steklov eigen value problem using finite element approximation, Computational Mathematics and Modelling 21 (2010) 109116.

[12] M. Kumar, A. Srivastava, A. Kumar Singh, Numerical simulation of singularly perturbed non-linear elliptic boundary value problems using finite element method. Applied Mathematics and Computation 219 (2012) 226236.

[13] El. M. Hssini, M. Massar, M. Talbi and N. Tsouli, Existence of solutions for a fourth order problem at resonance. Bol. Soc. Paran. Mat. (3s.) v. 322 (2014): 133142.SPM ISSN-2175-1188, ISSN-00378712 in press.

[14] P. Lindqvist, Notes on the p-Laplace equation, NO-7491 Trondheim, Norway August 2005.

[15] L. Li, L. Jin S. Fang, Existence and uniqueness of the solution to a coupled fractional diffusion system, a Springer open Journal, Advances in Difference Equations (2015) 2015:370.

[16] J.M. Neuberger, J.W. Swift, Newtons method and Morse index for semi-linear elliptic PDEs, International Journal of Bifurcation and Chaos 11 (2001) 801820.

[17] P. Sorin Ilias, P-laplacien Poids Indfini, Vol. LVIII, No. 2/2006.

[18] Z. Weng, S. Zhai, X. Feng, An improved two-grid finite element method for the Steklov eigenvalue problem. Applied Mathematical Modelling 39 (2015) 29622972.

[19] Y. Yang, Q. Li, S. Li Nonconforming finite element approximations of the Steklov eigenvalue problem. Applied Numerical Mathematics 59 (2009) 23882401.

[20] E. El-zahrani, H. Serag, Existence of Weak Solutions for Nonlinear Elliptic Systems on, Electron. J. Diff. Eqns., Vol. 2006, No. 69, 110, 2006. 